\title{
Cerebral venous thrombosis: An experience with anticoagulation with low molecular weight heparin
}

\author{
Lalitha V. Pillai, Dhananjay P. Ambike, Satish Nirhale, S. M. K. Husainy, Satish Pataskar
}

Cerebral venous sinus thrombosis [CVST] is often an infrequent cause of neurological dysfunction resulting in admissions in Intensive care units [ICU]. Because of its myriad presentation it may be under diagnosed. Unfractionated Heparin [UFH] has been advocated in treatment but needs frequent monitoring. We studied the clinical profile of patients of cerebral venous sinus thrombosis, use of low molecular weight heparin [LMWH] with emphasis on safety in 64 patients of CVST.

Key Words: Heparin, cerebral venous sinus thrombosis, low molecular weight heparin, oral contraceptive, homocysteine

\section{Introduction}

Cerebral venous sinus thrombosis (CVST) is often an under diagnosed cause for acute or slowly progressive neurological deficit. It was recognized for more than 150 years, but on autopsies and was therefore always considered lethal. ${ }^{[1]}$ CVST has wide spectrum of signs and symptoms, which evolve suddenly or over the weeks. It is clinically challenging and mimics practically all-neurological conditions such as, meningitis, encephalopathy benign intracranial hypertension, and stroke. It can affect all age groups with unpredictable outcome but it is not as lethal as presumed.

With increasing awareness of this entity and easy accessibility to non invasive tests like CT and MRI scans, CVST cases are now being diagnosed more frequently ${ }^{[2]}$ such that it is now thought to occur more commonly than previously assumed. It is also probably true that this condition is more frequent in the underdeveloped countries. ${ }^{[3]}$ The diverse presentation and unpredictable clinical outcome has lead to controversies in the management.

\section{From:}

Critical Care Department, Lokmanya Hospital, Chinchwad, Pune, India Correspondence:

Dr. Lalitha Pillai,

Director Critical Care Department, Lokmanya Hospitals, Chinchwad, Pune, Maharashtra - 411033, India. E-mail: Ialithapillai@rediffmail.com
Although heparin has been advocated in the treatment for the last 50 years, anticoagulation with dose-adjusted heparin has been strongly recommended after results of trails became available. An editorial in STROKE ${ }^{[1]}$ also remarked that placebo controlled trials of heparin was no longer justified. Heparin requires continuous intravenous administration with close laboratory monitoring making therapy difficult in a resource limited setting. Hence we decided to study the efficacy of LMWH in the treatment of CVST with special emphasis on its safety in presence of hemorrhagic infarcts. We report this series of 64 patients where anticoagulant treatment with therapeutic dose of LMWH did not cause any further deterioration in neurological status but improved the outcome in patients with CVST.

\section{Materials and Methods}

Our Hospital is situated in an industrial township with 27 bed ICU. We had a large number of cases suspected and diagnosed as "CVST". For ease of administration and monitoring LMWH was given to all these patients. During this period all patients of CVST were treated with therapeutic doses of LMWH subcutaneously from day one and along with oral anticoagulants from day three. LMWH was continued for an average of 7-10 days by 
which time INR was between 2.5-3.0. Reasons to stop medication were major extracranial hemorrhage, and/or symptomatic new intracranial hemorrhage confirmed by CT scan. All details were entered in a proforma.

A retrospective review of proformas of all cases of CVST treated between 1997 July and December 2004 was conducted. All 64 patients where diagnosis of CVST was confirmed by CT/MRI brain venogram were included in this study. All the patients were symptomatic, and had deficits, which were non-resolving or worsening. Hemorrhagic infarcts, associated mastoid infections and recent surgery were not a contraindication for treatment. One case with deep-seated hemorrhagic infarct, where diagnosis was doubtful, was not treated with LMWH. All patients had basic investigations such as haemogram, electrolytes, blood sugar levels, renal function tests and chest radiographs. Liver function tests, coagulation studies, inflammatory markers and homocysteine levels were done in selected patients because of financial constraints.

Patients were shifted out of intensive care unit when they improved symptomatically or were stable neurologically. On admission GCS and Sinus Venous Thrombosis (SVT) severity scale was entered on all records. Those patients of GCS $<8$ were ventilated. All the patients were given phenytoin in loading and maintenance doses, along with mannitol. Antibiotics were used as indicated by clinical situations. Patients were asked to follow-up in OPD on discharge.

\section{Results}

64 patients were studied during the period 1997 to 2004 of which 38 were males [59\%] and 26 were females [41\%]. Average age of patients was 32.27 [S.D. \pm 11.8 ] with no gender difference.

Risk factors were found in 36 [56.3\%] of the patients. In females 19 out of 26 [73.\%] had risk factors for development of CVST. Principally it was use of oral contraceptive pills [38.4\%], peurperium [23\%] and one case was of recent surgery. Two of the cases in peurperium period had LSCS done and both of them had mastoiditis. 11 out of 15 patients tested for homocysteine levels had raised levels of which 14 were males. [Table 1]

Presenting symptoms occurred over 1 to 20 days (av- erage 4.8 days) before admission. Headache was the presenting symptom in 53 [82.8\%] of the cases and headache with seizures was seen in 27 [42\%] cases. Constellation of symptoms of headache drowsiness and seizures appeared in 15 patients [23.4\%]. [Table 2]

55 cases had CT scan done, 46 cases had MRI done and in 39 cases CT scan was followed by MRI/ MRI angio. Eight cases on admission showed normal CT scan. Superior sagital sinus [SSS] was the only sinus involved in 25 cases. Superficial sinuses were involved in 36 cases, deep in 16 cases and 12 showed involvement of both group of sinuses. [Table 3] Parietal infarct was found in 16 cases while frontal and thalamic infarcts were found in six cases each.

8 patients were having GCS $<8$ and were ventilated while 56 were having GCS $>8$. There was no correlation found in duration of presenting symptoms and hospital stay. High sinuous venous thrombosis [SVT] score [Table 4] was significantly associated with low GCS as

\begin{tabular}{|c|c|c|c|}
\hline Risk factors & Total & Frequency & $\%$ \\
\hline OCP & 26 & 10 & 38.46 \\
\hline Delivery & 26 & 6 & 23.1 \\
\hline DVT & 64 & 5 & 7.8 \\
\hline Head injury & 64 & 1 & 1.56 \\
\hline Homocysteine & 15 & 11 & 73.3 \\
\hline Anti C A & 8 & 2 & 25 \\
\hline Mastoid & 64 & 5 & 7.81 \\
\hline
\end{tabular}

Table 2: Presenting symptoms and signs of patients with cerebral venous sinus thrombosis

\begin{tabular}{lcc}
\hline Symptoms & Total & Percent \\
Headache & 53 & 82.8 \\
Seizures & 36 & 56.2 \\
Loss of consciousness & 26 & 40.6 \\
Fever & 16 & 25 \\
Nausea/Vomiting & 34 & 53.1 \\
Ear Infection & 12 & 18.8 \\
Sinusitis & 9 & 14 \\
Any other infection & 11 & 17.2 \\
Giddiness/Vertigo & 23 & 35.9 \\
Hemiplegia & 36 & 56.3 \\
Visual Disturbances and papilloedema & 17 & 26.6 \\
CrN Palsy & 21 & 32.8 \\
\hline
\end{tabular}

Table 3: Type of venous sinus involved in study patients

\begin{tabular}{lcc}
\hline Type of sinus & Frequency & $\%$ \\
Superficial & 36 & 56.25 \\
Deep & 16 & 25 \\
Superficial + Deep & 12 & 18.75
\end{tabular}




\begin{tabular}{|c|c|c|c|}
\hline $\begin{array}{l}\text { Score } \\
1 .\end{array}$ & $\begin{array}{l}\text { Headache } \\
\text { Slight }\end{array}$ & $\begin{array}{l}\text { Focal signs } \\
\text { Transient minimum } \\
\text { or hyperasthesia }\end{array}$ & Seizure \\
\hline 2. & Moderate & Transient or slight paresis & \\
\hline 3. & Severe & Mild paresis & Seizure \\
\hline 4. & & Mod Paresis & Seizures status \\
\hline 5. & & Severe paresis or plegia & $\begin{array}{l}\text { Confused or } \\
\text { psychotic }\end{array}$ \\
\hline 6. & & & Somnolent \\
\hline 7. & & & Stupor \\
\hline 8. & & & Coma \\
\hline 9. & & & Dead \\
\hline
\end{tabular}

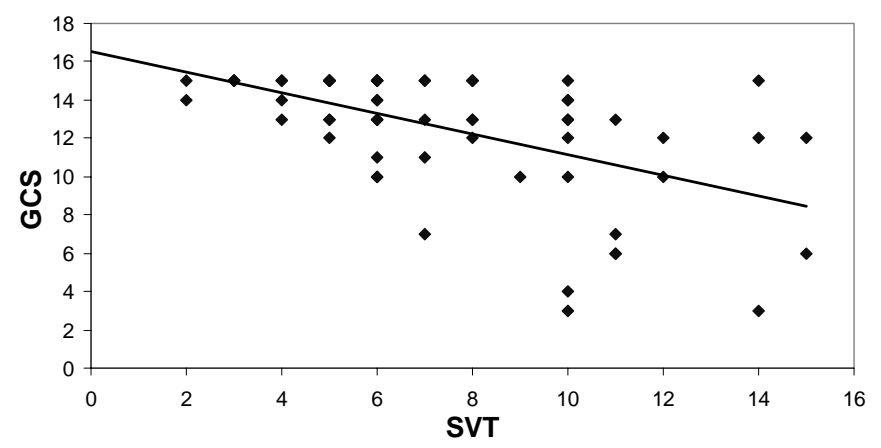

Figure 1: Scatter plot showing inverse relationship between GCS and SVT severity scale

per the scatter plot [Figure 1]. Average hospital stay was 10.6 days [s.d. \pm 7.48 ].

Repeat CT/MRI could be done in 17 patients prior to discharge. Only 2 patients showed complete resolution of thrombosis. Rest 15 showed partial recanalization though patients had good recovery. Persistent residual neurodeficit was found in three patients at discharge. We did not have any hemorrhagic complications in any of the patients.

Three patients who underwent neurosurgical decompression due to deterioration within 24 hours of admission were all women. In two of them LMWH was continued in postoperative period followed by oral anticoagulant and all three survived.

There were 4 deaths out of which two are males. One of them, a 21 year old male had mastoiditis, intracranial abscess and cerebellar infarct due to involvement of straight and transverse sinus. Other 42-year-old male had hyperhomocysteinemia with previous cortical and mesenteric vein thrombosis. Of the females who died, one was 71 years old and had anti-cardiolipin antibody test positive. Other was 50 years old with no risk factors. The rest of patients had good outcome. One pa- tient had dilated nonreacting pupils with absent doll's eye movements and remained comatose for more than a month. On discharge he was conscious but required assisted care, 6 months later he was ambulatory and did not required any support.

\section{Discussion}

CVST is not so rare but an alarming disease usually beginning with a severe headache and may lead to seizures, neurological deficits and even death. It has been reported to cause $10-20 \%$ of young strokes in India. ${ }^{[3,4]}$

No epidemiological data is available hence the exact incidence is debatable. The Dutch study of de Bruijn, $\mathrm{J}$ stam of CVST study group ${ }^{[5]}$ could recruit only 60 patients from 14 hospitals in Netherlands and U.K. between 1992 and 1996. Many other western studies also have a small number of patients or have been done over a large number of years. Most Indian studies have fairly large number of cases thereby suggesting that the incidence here is not as rare as previously thought of and should be considered in all cases of young stroke and neurological syndromes in appropriate setting. Like all other studies we also had similar demographic picture. The average age in our series was 32.27 years. Predisposing risk factors has been identified in $80 \%$ patients in some series. Prothrombotic states ${ }^{[6]}$ such as factor $\mathrm{V}$ leiden mutation, protein $\mathrm{C}$ or $\mathrm{S}$ deficiency, antithrombing 111, pregnancy and puerperium are regarded as risk factors but in many cases the cause remains unknown (20-25\%). In the absence of risk factors patients may need close follow-up and may require lifelong anticoagulations. $43.75 \%$ of cases in our series had no risk factor. Post gestational and post puerperal CVST has been described more often from Indian subcontinent with post puerperal occurring more commonly but use of oral contraceptive (OC) pills [38.4\%] in females was a major risk factor with peurperium coming second in our study in this industrial township. In developed countries use of oral contraceptives has been identified as a major risk. A Dutch study showed age adjusted odds ratio of 13 for oral contraceptive use ${ }^{[5]}$ and CVST. The higher percentage of oral contraceptive use [38.4\%] as a risk factor in our series of 26 female cases suggests a western trend. This should warn about the possibility of indiscriminate use of $\mathrm{OC}$ pills. Hyperhomocysteinemia was also found in $73.3 \%$ of tested patients and hence appears to be a significant modifiable risk factor. 
The extreme diversity in CVST clinical presentation makes it a challenge for both diagnosis and treatment. In all major series, as also in ours, headache [82.8\%] was the main presenting symptom, followed by seizures [56.2\%]. Variability of the clinical signs can be explained by the extensive collateral circulation within cerebral venous system, which allows for compensation in early stages of venous occlusion. ${ }^{[7]}$ The small cerebral veins drain into large veins such as vein of Galen. These bigger veins empty into dural sinuses, which drain into Internal Jugular vein. The venous territories are less well defined than the arterial territories because of extensive anaestomosis between cortical veins. Moreover there is a continuing process in which balance of prothrombotic and thrombolytic processes is disturbed leading to progression of venous thrombosis with time, resulting in dilated venous and capillary bed, development of interstitial edema, increased cerebrospinal fluid [CSF] production, decreased CSF absorption and rupture of venous structures (hematoma). The slow growth of thrombus and good collateralization explains the usually gradual onset of symptoms. Most patients in our series recovered completely and this was unrelated to duration of presenting symptoms and signs. Obstruction of sinuses cause raised ICT because of obstructed CSF drainage leading to papilloedema and impaired vision seen in 17 of our cases.

The focus of investigation should be on establishing diagnosis and finding an underlying cause. CT scan is first imaging modality because of its availability. A radiologist has been usually the first person to alert the possibility of CVST. In $20 \%$ cases it can be normal but it can also exclude other causes such as intracranial bleed, space-occupying lesion. The opacification of collateral veins, present in $10-20 \%$ of cases, in wall of sagital sinus after contrast injection is the empty delta sign on CT. Presence of hemorrhagic infarcts is also suggestive of CVST. MRI or MR venography has replaced angiography as gold standard ${ }^{[1]}$ in diagnosis of CVST. These modalities also pick up infections such as mastoiditis and sinusitis. CT venography has been reported to be superior to MRV. CSF examinations are not pathognomic but usually show increased pressure, proteins, RBCs and pleocytosis and rules out subarachnoid hemorrhage, meningitis in appropriate clinical setups.

Current treatment options for CVST include anti-throm- botic therapy with UFH, LMWH, oral anticoagulants, intravenous thrombolysis with urokinase, rtPA, local thrombolysis by selective sinus catheterization and a combination of thrombolysis and anticoagulation ${ }^{[4]}$ in addition to symptomatic therapy.

Considering the high prevalence of CVST in developing countries, the treatment options should be determined by easy availability, less intensive monitoring, cost effective, associated with less complications and most important, clinically effective.

A number of individual case reports, uncontrolled series retrospective studies and randomized trials ${ }^{[8-10]}$ have well documented the efficacy and safety of heparin in CVST, even in presence of hemorrhagic conditions. ${ }^{[4]}$ Diaz et al reviewed 203 CVST cases between 1942 and 1990 and compared the outcome in patients treated with and without heparin. $91 \%$ survived in first group compared to $36 \%$ in the second. ${ }^{[11]}$ The target APTT time was 80-100 seconds with respect to neurological recovery. Neonatal CVST is the only situation in which heparin has not been shown to improve the outcome and most authors do not recommend its use in this situation. ${ }^{[4]}$ Heparin though relatively inexpensive has been advocated with continuous intravenous infusion and needs close laboratory monitoring which may not be widely available and offsets this advantage.

Low molecular weight heparin has also been found to be equally effective. ${ }^{[5,12]} \mathrm{LMWH}$ is derived from UFH but differ in mode of action. It preferably inhibits factor $X_{a}$ rather that thrombin and has higher bioavailability, longer half-life, reduced protein binding and dose independent clearance. They have more predictable anticoagulant response and can be given subcutaneously without monitoring. There is less antiplatelet activity and vascular permeability resulting in less bleeding as compared to UFH. Data is not available which allow direct comparison of LMWH versus UFH in treatment of CVST. Randomized trials in patients with leg vein thrombosis or pulmonary embolism show that low-molecular-weight heparins, are as effective as UFH and cause fewer hemorrhagic complications ${ }^{[13-15]}$

As, we found it difficult to monitor heparin therapy we started using LMWH in 1997 onwards and as, there was improvement without any complications we continued 
to use it in all our patients. There was no new symptomatic cerebral hemorrhages in our study, which is significant considering high rate of venous hemorrhagic infarcts [78\%]. Three patients, all females, needed neurosurgical intervention. As all of them needed it within 24 hours of admission we feel that this may not be related to complication of LMWH but due to the natural course of the disease. In two of them LMWH was continued in postoperative period followed by oral anticoagulant and all three survived. If the patient deteriorates despite adequate heparinisation or presents moribund with coma, selective catheter-guided local thrombolysis may be an option, inspite of the increased haemorrhagic risk. ${ }^{[16]}$ Surgical decompressive craniotomy, thrombectomy and evacuation of intracerebral haematoma need still further evaluation before it is recommended as a safe procedure. There were no confirmed thromboembolic complications either, the prevention of which is probably an important benefit of anticoagulant treatment in patients with sinus thrombosis. ${ }^{[11]}$ The Dutch double blind placebo study used LMWH for 3 weeks in the treatment arm, this was then followed by oral anticoagulants. Our study significantly differed from them in that we used LMWH and started oral anticoagulants from third day onwards thereby reducing the duration of LMWH in our study by one third. This saved cost and also reduced the hospital stay with compromising on safety and efficacy. We suggest that a multicentric randomized control study of LMWH and UFH be conducted to compare benefits and complications of both the drugs. Most studies have used higher doses of intravenous heparin to maintain APTT 2.5 times the control but in puerperal CVST 5000 units 8 hourly subcutaneous has been considered safe and effective. ${ }^{[4]}$ There is room for modification of the dose in other conditions too, making it an attractive therapeutic option. As it stands till date LMWH with its ease of administration and advantage of not requiring laboratory monitoring warrants its use in most ICUs and peripheral centers.

\section{Conclusion}

Due to its varied presentation CVST should be considered in all cases of severe headache, seizures and young stroke and neuroimaging should be asked for. Gold standard for diagnosis of CVST is MRI. MR angio is indicated in early [ $<5$ days] or late stages [ $>6$ weeks] or whenever MRI shows equivocal signal. Homocysteine levels should be measured in all cases of venous throm- bosis as it can be easily corrected. Based on our results and previous randomized data we conclude that anticoagulant treatment with LMWH is safe and effective in patients with sinus thrombosis even in those with intracranial hemorrhage.

\section{References}

1. Bousser MG, Cerebral venous thrombosis; nothing, heparin, or local thrombolysis? Stroke 1999;30:481-3.

2. Allroggen $\mathrm{H}$, Abbott RJ. Cerebral venous sinus thrombosis Postgrad Med J 2000;76;12-5.

3. Srinivasan K. Ischemic cerebrovasular disease in the young: Two common causes in India. Stroke 1984;15;733-5.

4. Nagaraja D, Sarma GR. Treatment of cerebral sinus/venous thrombosis. Neurol India 2002;50;2:114-6.

5. de Bruijn SF, Stam J. For the CVST Study Group. Randomized, placebo controlled trial of anticoagulant treatment with low-molecular-weight heparin for cerebral sinus thrombosis. Stroke 1999;30:484-8

6. Deschiens M. Coagulation studies, factor V leiden, and anticadiolipin antibodies in 40 cases of cerebral venous thrombosis. Stroke 1996;27;1724-30.

7. Schaller B, Graf R. Cerebral Venous Infarction: The Pathophysiological Concept. Cerebrovasc Dis 2004;18:179-88.

8. Bousser MG, Chiras J, Sauron B, et al. Cerebral venous thrombosis. A review of 38 cases. Stroke 1985;116:199-213.

9. Halpern JP, Morris JG, Driscoll GL. Anticoagulants and cerebral venous thrombosis. Aust N Z J Med 1984;14:643-8.

10. Nagaraja D, Taly AB, Haridas VT, et al. Heparin in hemorrhagic infarction in cerebral venous sinus thrombosis. J Asso phys India 1998;46:706-7.

11. Diaz JM, Schiffman JS, Urban ES, Maccario M. Superior sagittal sinus thrombosis and pulmonary embolism: A syndrome rediscovered. Acta Neurol Scand 1992;86:390-6.

12. Prakash C, Bansal BC. Cerebral venous thrombosis. J Indian Acad Clin Med 2000;5:55-61.

13. Lensing AWA, Prins MH, Davidson BL, Hirsh J. Treatment of deep venous thrombosis with low-molecular-weight heparins: $A$ meta-analysis. Arch Intern Med 1995;155;601-7.

14. Hersh J, Siragusa S, Cosmic B, Ginsberg JS. Low molecular weight heparins $[\mathrm{LMWH}]$ in the treatment of patients with acute venous thromboembolism. Thromb Haemost 1995;74:360-3.

15. Low-molecular-weight heparin in the treatment of patients with venous thromboembolism: The Columbus investigators. N Engl J Med 1997;337:657-62.

16. Fray JI, Muro GJ, Mcdougall CG, et al. Cerebral venous thrombosis - combined intrathrombus rtpa and intravenous heparin. Stroke 1999;30:489-94. 